\title{
On the Normalization of Meson Eigenstates in Functional Quantum Theory of the Nonlinear Spinor Field
}

\author{
D. Englert and H. Stumpf \\ Institut für Theoretische Physik der Universität Tübingen
}

(Z. Naturforsch. 29 a, 376-384 [1974] ; received 13 November 1973)

\begin{abstract}
In the framework of the functional quantum theory of the nonlinear spinor field the normalization of functional eigenstates of scalar mesons is performed. By calculating the lowest approximation of the functional eigenstates for scalar mesons from the dynamical functional equation of the theory, it follows that scalar mesons of positive as well as of negative parity are admitted. Requiring a positive norm for physical particles the result of normalization shows that mesons with positive parity are excluded, while meson states with negative parity give a positive definite norm. This corresponds to experimental experience.
\end{abstract}

Nonlinear spinor theory with noncanonical relativistic Heisenberg quantization treated by Heisenberg and coworkers is an approach to a unified microscopic description of matter ${ }^{1,2}$. To obtain a meaningful physical information by this theory at least the socalled global observables have to be calculated. As the spinorfield operators $\Psi_{\alpha}(x)$ cannot be identified with a special free matter field of conventional quantum field theories the methods of construction of observables provided these theories do not suffice to solve this task correctly for the nonlinear spinor field. This has been shown in detail in ${ }^{3}$ and has been ignored so far. To remove this difficulty Stumpf proposed the introduction of a functional quantum theory 4,5 which has been prepared in a series of papers by Stumpf and coworkers. The essential point of this approach is a map of the ordinary Hilbert space of quantum field theory into a corresponding functional Hilbert space, where the observables of interest have to be calculated. To do this the functional Hilbert space has to be equiped with a suitable relativistic invariant functional scalar product, which has been proposed in a preceding paper ${ }^{6}$. To show its validity it has to be tested first for cases which are already solved conventionally. This has been done successfully for generalized free fields by Englert $^{7}$ and for the lowest order of the interaction representation of a selfcoupled fermion field by Illig ${ }^{8}$. In this paper this method shall be applied now to a problem of nonlinear spinor theory itself, namely the normalization of functional meson states, which is required for

Reprint requests to Prof. Dr. H. Stumpf, Institut für Theoret. Physik der Universität Tïbingen, D-7400 Tübingen, Auf der Morgenstelle 14. subsequent calculations of crossections. A first investigation on this problem has been done by Schäfer ${ }^{9}$. By using the Weyl representation of the nonlinear spinor field introduced by Dürr ${ }^{10}$, his boson norm diverges. But this gives no argument against functional normalization. It rather shows, that functional normalization is very sensible against inconsistencies of the theory. In this case the inconsistency is due to the fact, that intermediate and real states of the theory which occur in functional normalization procedure are not massless. But then one is not allowed to normalize them by scalarproducts for massless fermions as the Weyl representation requires. By this argument follows, that the Weyl representation of the nonlinear spinor field has to be rejected. Therefore we use in this paper the original Dirac representation of the theory, which can be justified also by cosmological considerations. As will be demonstrated in the following, the results are finite and physically meaningful. A normalization of the boson amplitudes which is equivalent to the Bethe-Salpeter normalization procedure has been performed already by Heisenberg ${ }^{1}$. But so far the Bethe-Salpeter normalization is restricted to the bound states of the twopoint function and no suitable generalization is known. So the advantage of the procedure given here lies not in this special problem, but in its intention of generality. In this sense this contribution has to be considered to be an illustration of the general functional approach to quantum theory of high energy phenomena. As we use the lowest approximation of the functional dy. namical equation to calculate the meson eigenstates only scalar mesonstates can be derived. So we restrict ourselves in the following to the normalization of those states. The treatment of vector meson states 
can be done on the same pattern and will be performed in further papers.

In this paper we have adopted the Einstein summation convention, i. e. summation respective integration over variables occuring twice.

Some remarks have still to be made about the meaning of the indices. We use super-indices in $\lambda$-isospin- and spin-space. For convenience we do not distinguish the indices referring to the different subspaces, as the symbol of the eigenfunctions do suffice to specify the space in consideration.

\section{Fundamentals}

In functional quantum field theory, we describe the physical states by the time ordered functionals

$$
\begin{aligned}
& |\mathfrak{I}(j, a)\rangle=\sum_{n=0}^{\infty} \frac{i^{n}}{n !} \\
& \cdot \tau_{n}\left(x_{1} \ldots x_{n} \mid a\right) j^{\alpha_{1}}\left(x_{1}\right) \ldots j^{x_{n}}\left(x_{n}\right)\left|\varphi_{0}\right\rangle . \\
& \alpha_{1} \ldots \alpha_{n}
\end{aligned}
$$

The basic assumptions and the definitions can be found in a review article in ${ }^{3}$.

Time ordering of the field operators can be defined in an invariant manner by ${ }^{6}$ :

$$
\begin{aligned}
& \tau_{n}\left(x_{1} \ldots x_{n} \mid a\right):=\left\langle 0\left|T \Psi_{\alpha_{1}}\left(x_{1}\right) \ldots \Psi_{\alpha_{n}}\left(x_{n}\right)\right| a\right\rangle \\
& \quad \alpha_{1} \ldots \alpha_{n} \\
& :=\sum_{\lambda_{1} \ldots \lambda_{n}}(-1)^{\mathrm{P}}\langle 0| \Psi_{\alpha / \lambda_{1}}\left(x_{\lambda_{1}}\right) \ldots \Psi_{\alpha / \lambda_{n}} \\
& \quad \cdot\left(x_{\lambda_{n}}\right)|a\rangle \Theta\left[n\left(x_{\lambda_{1}}-x_{\lambda_{2}}\right)\right] \ldots \Theta\left[n\left(x_{\lambda_{n-1}}-x_{\lambda_{n}}\right)\right] .
\end{aligned}
$$

$n$ is a timelike unit vector with $n^{2}=1$ and $n^{0}>0$. Additionally we have to derive the spectral representation of the $\tau$-functions in momentum space, as the physical scalar product is defined in terms of it. In ${ }^{6}$ this was done by choosing the special vector $n=$ $(1,0,0,0)$. But thereby a special frame of reference was selected and this caused a loss of invariance in the formulas. To obtain an explicitly invariant representation the same calculations can be done without great complications using a general vector $n$. Later we shall specialise this vector again by identifying it with a unit vector in the direction of the total momentum of the system to be considered, which is despite of the specialisation an invariant procedure, too.

To begin with every vector can be decomposed in a component parallel to $n$ and the orthogonal rest ${ }^{11}$

$$
x^{u}=(n x) n^{\mu}+\boldsymbol{x}^{\mu} .
$$

The vectors $\boldsymbol{x}^{\mu}$ are situated in a three dimensional subspace of Minkowski space. The integration mea- sure $\mathrm{d}^{4} x$ then can be written $\mathrm{d}^{1}(n x) \mathrm{d}^{3} \boldsymbol{x}$. By applying the invariant decomposition of the $\delta$-function

$$
\delta^{4}(x)=\delta(n x) \delta^{3}(\boldsymbol{x})
$$

we get in complete analogy to ${ }^{6}$ the spectral representation:

$$
\begin{aligned}
& \tau_{n}\left(q_{1} \ldots q_{n} \mid \mu_{n}\right) \\
& \quad \alpha_{1} \ldots \alpha_{n-1} \\
& =\sum_{\mu_{1} \ldots \mu_{n-1}} \sum_{\lambda_{1} \ldots \lambda_{n}}(-1)^{\mathrm{P}} M\left(\mu_{1} \ldots \mu_{n}\right) \\
& \alpha_{\lambda_{1}} \ldots \alpha_{\lambda_{n}} \\
& \cdot\left(\frac{i}{2 \pi}\right)^{n-1}(2 \pi)^{4 n} \delta\left(p_{\mu_{n}}-\sum_{s=1}^{n} q_{\lambda_{s}}\right) \\
& \quad \cdot \prod_{s=1}^{n-1}\left\{\delta\left(\boldsymbol{p}_{\lambda_{n}}-\sum_{r=1}^{s} \boldsymbol{q}_{\lambda_{r}}\right)\left(\sum_{r=1}^{s} q_{\lambda_{r}} n-p_{\mu_{s}} n+i \varepsilon\right)^{-1}\right\}
\end{aligned}
$$

with

$$
\begin{aligned}
& M\left(\mu_{1} \ldots \mu_{n}\right):=\left\langle 0\left|\Psi_{\alpha_{1}}(0)\right| \mu_{1}\right\rangle \ldots \\
& \alpha_{1} \ldots \alpha_{n} \\
& \cdot\left\langle\mu_{n-1}\left|\Psi_{\alpha_{n}}(0)\right| \mu_{n}\right\rangle .
\end{aligned}
$$

Formula (1.5) is the spectral representation, which will be used later to calculate the scalar product. The time ordering in the twopoint- and Green'sfunctions, which we need in the next section, have to be defined in an invariant manner too. This is done in the Appendix I and results in a new formula, slightly different from the others, used until now.

\section{Eigenstate Equations of Mesons}

In order to obtain a meson wave function, we start with the nonlinear spinor equation in its functional formulation ${ }^{3}$ :

$$
\begin{gathered}
\left\{D_{\alpha}^{\beta} \partial_{\beta}+V_{a}^{\beta \gamma \delta}\left[\partial_{\beta} \partial_{\gamma} \partial_{\delta}-3 F_{\beta \gamma} \partial_{\delta}\right]-i \varrho_{0} j_{\alpha}\right\} \\
\cdot|\mathfrak{I}(j, a)\rangle=0 .
\end{gathered}
$$

For brevity, we have absorbed the space-time variable in the spinorial index as is usually done.

$D_{\alpha}^{\beta}$ is the Dirac operator in the Hermitean representation, which we use here in the notation of Dürr and Wagner ${ }^{10}$.

$$
\begin{gathered}
D_{a}^{\beta}=i \Gamma_{a}{ }^{\nu \beta} \delta\left(x_{\alpha}-\mathrm{x}_{\beta}\right) \partial / \partial x_{\beta}{ }^{\nu}, \\
\Gamma_{a}{ }^{\nu \beta}=-\left(\lambda_{2}^{\prime} \beta \gamma^{\prime}\right)_{\alpha}{ }^{\beta} .
\end{gathered}
$$

The Vertex operator is local, real and completely antisymmetric:

$$
V_{\alpha \beta \gamma \delta}=\left(l^{2} / 2\right) \delta\left(x_{\beta}-x_{\alpha}\right) \delta\left(x_{\gamma}-x_{\alpha}\right) \delta\left(x_{\delta}-x_{\alpha}\right) \bar{V}_{\alpha \beta \gamma \delta} .
$$


With the normal transformation

$$
|\mathfrak{I}(j)\rangle=\exp \left\{-\frac{1}{2} j_{\alpha} F^{\alpha \beta} j_{\beta}\right\}|\Phi(j)\rangle
$$

we get from (2.1) an equation for the $\Phi$-functional. Our solution procedure starts by the application of the causal Green's function, which inverts the Dirac operator $D_{a}^{\beta}$.

By this the equation

$$
\partial_{*}|\Phi(j)\rangle=\mathbf{O}_{*}(j, \partial)|\Phi(j)\rangle
$$

results, with the following functional operator

$$
\begin{aligned}
\mathbf{O}_{\varkappa}(j, \partial):= & -G_{\varkappa}{ }^{\alpha} V_{\alpha}{ }^{\beta \gamma \delta}\left[d_{\beta} d_{\gamma} d_{\delta}-3 F_{\beta \gamma} d_{\delta}\right] \\
& -F_{\%}{ }^{1 \alpha} j_{\alpha}
\end{aligned}
$$

and the definitions

$$
d_{x}:=\partial_{\varkappa}-F_{\gamma^{\alpha}} j_{\alpha}
$$

and

$$
F_{\varkappa}{ }^{1 \alpha}:=-F_{\varkappa}{ }^{\alpha}-i \varrho_{0} G_{\varkappa}{ }^{\alpha} .
$$

Due to noncanonical quantization we have $\varrho_{0}=0$. Equation (2.6) singles out one coordinate. As the $\varphi$-functions are antisymmetric, the corresponding equation has to be invariant against permutation of the coordinates. This can be done by applying a symmetrization operator $\mathbf{S}_{\varkappa}$ to the equation.

Here we choose the symmetrization by functional integration, which has been proposed in a paper of Dammeier ${ }^{12}$, where the details of this functional integral and some references are given.

So we get the equation

$$
\begin{aligned}
\int \delta j^{*} \partial_{\varkappa}|\Phi(j)\rangle & =|\Phi(j)\rangle=\int \delta j^{*} \mathbf{O}_{\varkappa}(j, \partial)|\Phi(j)\rangle \\
& =: \mathbf{O}(j, \partial)|\Phi(j)\rangle .
\end{aligned}
$$

This homogenous equation for the functional $|\Phi(j)\rangle$ has to be solved now. We shall do this following the methods given by Stumpf ${ }^{3}$.

Applying the projection operators

$$
\mathbf{P}_{n}:=n !\left|D_{n \alpha_{1} \ldots \alpha_{n}}\right\rangle\left\langle D_{n}^{\alpha_{1} \ldots \alpha_{n}}\right| ; \Pi_{n}:=\sum_{v=n+1}^{\infty} \mathbf{P}_{v}
$$

the state functional $|\Phi(j)\rangle$ can be decomposed into

$$
|\Phi(j)\rangle=\mathbf{P}_{\varrho}|\Phi(j)\rangle+\Pi_{\varrho}|\Phi(j)\rangle
$$

where $\varrho$ is the smallest number for which we have $\mathbf{P}_{Q}|\Phi(j)\rangle \neq 0$. This number is fixed by the symmetries and quantum numbers of the eigenstates to be considered. Defining

$$
\left|\Phi_{Q}(j)\right\rangle:=\mathbf{P}_{Q}|\Phi(j)\rangle=i^{\varrho} \varphi_{Q \alpha_{1} \ldots \alpha_{n}} \mid D_{Q}^{\left.a_{1} \ldots a_{n}\right\rangle}
$$

an equation for $\left|\Phi_{\rho}(j)\right\rangle$ alone can be derived from (2.10). By using a Neumann series expansion for the elimination of $\Pi_{\varrho}|\Phi(j)\rangle$ from (2.10) we obtain the equation ${ }^{3}$ :

$$
\left|\Phi_{Q}(j)\right\rangle=\mathbf{P}_{e} \mathbf{O}(j, \partial) \sum_{v=0}^{\infty}\left[\Pi_{e} \mathbf{O}(j, \partial)\right]^{\nu}\left|\Phi_{Q}(j)\right\rangle .
$$

We use this equation to calculate the function $\varphi_{Q}$ in a first approximation by taking into account only the first term of the sum. As we deal with mesons, we have $\varrho=2$. Calculating the projection of that approximate equation on the power functional $\left|D_{2}\right\rangle$ we can derive an equation for the $\varphi_{2}$-function.

$$
\left\langle D_{2} \mid \Phi_{2}\right\rangle=\left\langle D_{2}\left|\mathbf{P}_{2} \mathbf{O}(j, \partial)\right| \Phi_{2}\right\rangle .
$$

Using the commutation relations of the functional sources and their adjoint operators and observing the antisymmetry of the wave function we obtain after an elementary calculation the equation

$$
\varphi_{\alpha_{1} \alpha_{2}}=\frac{3}{2}\left[G_{\alpha_{1} \alpha} F_{\beta \alpha_{2}}-G_{\alpha_{2} \alpha} F_{\beta \alpha_{1}}\right] V^{\alpha \beta \gamma \delta} \varphi_{\delta \gamma} .
$$

Now we write down explicitly the space-time variables and with the vertex (2.4) equation (2.16) reads

$$
\begin{array}{r}
\varphi_{\alpha_{1} \alpha_{2}}\left(x_{1} x_{2}\right)=\frac{3}{2}\left[G_{\alpha_{1} \alpha}\left(x_{1} x\right) F_{\beta \alpha_{2}}\left(x x_{2}\right)-G_{\alpha_{2} \alpha}\left(x_{2} x\right)\right. \\
\left.\cdot F_{\beta \alpha_{1}}\left(x x_{1}\right)\right]\left(l^{2} / 2\right) \overline{V^{\alpha \beta \gamma \delta}} \varphi_{\delta \gamma}(x x) \cdot(2.17)
\end{array}
$$

The center of mass motion can be separated giving so

$$
\varphi_{\alpha_{1} \alpha_{2}}\left(x_{1} x_{2}\right)=\exp \left\{-i \frac{1}{2} J\left(x_{1}+x_{2}\right)\right\} \varphi_{\alpha_{1} \alpha_{2}}\left(x_{1}-x_{2}\right)
$$

where $J$ is the total momentum of the meson.

The following calculations are done in momentum space because the physical scalar product is given in momentum space too.

The Fourier transformed equation reads

$$
\begin{aligned}
& \tilde{\varphi}_{a_{1} \alpha_{2}}\left(q_{1} q_{2}\right)=\frac{3}{2}\left[\tilde{G}_{\alpha_{1} \alpha}\left(q_{1}\right) \tilde{F}_{\beta \alpha_{2}}\left(-q_{2}\right)\right. \\
& \left.-\tilde{G}_{\alpha_{2} \alpha}\left(q_{2}\right) \tilde{F}_{\beta \alpha_{1}}\left(-q_{1}\right)\right] \times \delta\left(q_{1}+q_{2}-J\right) \frac{l^{2}}{2} \bar{V}^{\alpha \beta \gamma \delta} \tilde{\varphi}_{\delta \gamma}
\end{aligned}
$$

with the definition

$$
\boldsymbol{\varphi}_{\delta \gamma}:=\int \mathrm{d} p \tilde{\varphi}_{\delta \gamma}(p)=(2 \pi)^{4} \varphi_{\delta \gamma}(0) .
$$

\section{Eigenstates of Mesons}

In order to calculate the meson eigenstates we have to expand the function in baryon number isospin and spin eigenfunctions. 
The mesons have baryon number $B=0$, so the expansion of the wave function reads

$$
\varphi_{\alpha_{1} \alpha_{2}}^{B=0}=\lambda_{\alpha_{1}}^{\mathrm{F}} \lambda_{\alpha_{2}}^{\mathrm{A}} \varphi_{\alpha_{1} \alpha_{2}}^{\mathrm{FA}}+\lambda_{\alpha_{1}}^{\mathrm{A}} \lambda_{\alpha_{2}}^{\mathrm{F}} \varphi_{\alpha_{1} \alpha_{2}}^{\mathrm{AF}} .
$$

The $\lambda_{\alpha_{1}}^{\mathrm{F}}$ and $\lambda_{\alpha_{2}}^{\mathrm{A}}$ are the eigenvectors of the baryon number operator in $\lambda$-space with eigenvalues +1 (fermion) and -1 (antifermion) respectively.

From the antisymmetry property of $\varphi_{\alpha_{1} \alpha_{2}}$ we can derive a relation for the expansion coefficients in (4.1) :

$$
\varphi_{\alpha_{1} \alpha_{2}}^{\mathrm{FA}}=-\varphi_{\alpha_{2} \alpha_{1}}^{\mathrm{AF}} .
$$

Using it we can simplify the corresponding equation.

Now we write down the vertex of Dürr and Wagner ${ }^{10}$ :

$$
\bar{V}_{\alpha \beta \gamma \delta}=\frac{1}{12}\left\{\beta \beta\left(I I+\gamma_{5} \gamma_{5}\right)\left[\left(3+\lambda_{2}{ }^{\prime} \lambda_{2}{ }^{\prime} \boldsymbol{\tau}^{\prime} \boldsymbol{\tau}^{\prime}\right) \gamma_{\mu}{ }^{\prime} \gamma^{\prime \mu}\right]\right.
$$$$
\left.+\left(I I+\gamma_{5} \gamma_{5}\right)\left[2\left(\lambda_{1}^{\prime} \lambda_{1}^{\prime}+\lambda_{3}{ }^{\prime} \lambda_{3}{ }^{\prime}\right) \boldsymbol{\tau}^{\prime} \boldsymbol{\tau}^{\prime} C C\right]\right\}_{\alpha \beta \gamma \delta} \text {. }
$$

Applying this vertex to the wave function (3.1) we obtain by observing relation (3.2) :

$$
\begin{aligned}
& \bar{V}^{\alpha \beta \gamma \delta} \boldsymbol{\varphi}_{\delta \gamma}=\frac{1}{6} i\left\{3\left(\beta \gamma_{\mu}{ }^{\prime}\right)^{\alpha \beta}\left(\beta \gamma^{\mu}\right)^{\gamma \delta}\right. \\
& +3\left(\beta \gamma_{5} \gamma_{\mu}^{\prime}\right)^{\alpha \beta}\left(\beta \gamma_{5} \gamma^{\mu}\right)^{\gamma \delta}-\left(\beta \lambda_{2}{ }^{\prime} \boldsymbol{\tau}^{\prime} \gamma_{\mu}{ }^{\prime}\right)^{\alpha \beta}\left(\beta \boldsymbol{\tau} \gamma^{\mu}\right)^{\gamma \delta} \\
& \left.-\left(\beta \gamma_{5} \lambda_{2}^{\prime} \boldsymbol{\tau}^{\prime} \gamma_{\mu}^{\prime}\right)^{\alpha \beta}\left(\beta \boldsymbol{\tau} \gamma_{5} \gamma^{\mu}\right)^{\gamma \delta}\right\} \boldsymbol{\varphi}_{\delta \gamma}^{\mathrm{FA}} .
\end{aligned}
$$

The expansion in isospin space can be done by the well known $\tau$-algebra. It reads

$$
\varphi_{\delta \gamma}^{\mathrm{FA}}=\tau_{\delta \gamma}^{0} \varphi_{\delta \gamma}^{\mathrm{FA}, 0}+\sum_{l=1}^{3} \tau_{\delta \gamma}^{l} \varphi_{\delta \gamma}^{\mathrm{FA}, l} .
$$

The last step is the decomposition in spin space. We expand the wave function in the $\Gamma$-algebra of Dirac spin, which consists of sixteen components. The vertex (3.3) describes the $v$-a-coupling scheme and therefore only eight of the sixteen components give a contribution to the wave function.

$\boldsymbol{\varphi}_{\delta \gamma}^{\mathrm{FA}},\left(\begin{array}{l}0 \\ l\end{array}\right)=\left(\gamma^{v} \beta\right)_{\delta \gamma} \boldsymbol{\varphi}_{v}^{\mathrm{FA},\left(\begin{array}{l}0 \\ l\end{array}\right), \mathbf{v}}+\left(\gamma^{v} \gamma_{5} \beta\right)_{\delta \gamma} \boldsymbol{\varphi}_{v}^{\mathrm{FA},\left(\begin{array}{l}0 \\ l\end{array}\right), \mathrm{A}}$.

For mesons with spin zero the expansion coefficients in (3.6) can be written in the following manner ${ }^{1}$ :

$$
\begin{aligned}
& \varphi_{v}^{\mathrm{FA},\left(\begin{array}{l}
0 \\
l
\end{array}\right), \mathrm{v}}=J_{v} C^{\left(\begin{array}{l}
0 \\
l
\end{array}\right), \mathrm{v}}, \\
& \boldsymbol{\varphi}_{v}^{\mathrm{FA},\left(\begin{array}{l}
0 \\
l
\end{array}\right), \mathrm{A}}=J_{v} C^{\left(\begin{array}{l}
0 \\
l
\end{array}\right), \mathrm{A}}
\end{aligned}
$$

where the vector part is given by the total momentum $J$ of the particle and there are left only scalar constants. Substituting all these relations into the wave Eq. (2.19) we obtain after a lengthy calculation the equation of the meson functions with baryon number $B=0$, Isospin $I, I_{3}$ and spin zero:

$$
\begin{aligned}
\tilde{\varphi}_{\alpha_{1} \alpha_{2}}^{0, \mathrm{II} 3}\left(q_{1}\right. & \left.q_{2}\right)=i l^{2}[3-I(I+1)] \sum_{\lambda_{1} \lambda_{2}}(-1)^{\mathrm{P}} \\
& \cdot \tilde{G}_{\alpha_{\lambda_{1} \alpha}}\left(q_{\lambda_{1}}\right) \tilde{F_{\beta \alpha_{\lambda_{2}}}}\left(-q_{\lambda_{2}}\right) \delta\left(q_{1}+q_{2}-J\right) \\
& \cdot\left\{-\left[\beta \lambda_{2}^{\prime} \tau^{\prime}\left(I, I_{3}\right) \gamma^{\prime \nu}\right]^{\alpha \beta} J_{\nu} C^{I I_{3}, \mathrm{~V}}\right. \\
- & {\left.\left[\beta \gamma_{5} \lambda_{2}^{\prime} \tau^{\prime}\left(I, I_{3}\right) \gamma^{\prime \nu}\right]^{\alpha \beta} J_{\nu} C^{I I_{3}, \mathrm{~A}}\right\} . }
\end{aligned}
$$

Meson eigenstates with spin unequal zero cannot be treated physically meaningful by Equation (2.17). For their treatment a higher approximation of (3.14) is required. So we restrict ourselves to the discussion of the simplest case, namely the spin zero mesons.

Finally the meson eigenstates will be given in their spectral representation. Thereby we need the functions $F$ and $G$ in their invariant definition of time ordering discussed in Section 2. From Appen$\operatorname{dix} I$ we get

$$
\begin{aligned}
& \tilde{F}_{\alpha \beta}(q)=\int \varrho_{1}\left(m^{2}\right) \mathrm{d} m^{2} \frac{i}{2 \sqrt{m^{2}-\boldsymbol{q}^{2}}}\left[\frac{q_{\mu}}{n q-\sqrt{m^{2}-\boldsymbol{q}^{2}+i \varepsilon}}\left(-\lambda_{2}^{\prime} \gamma^{\prime \mu} \beta\right)_{\alpha \beta}-\frac{q_{\mu}}{n q+\sqrt{m^{2}-\boldsymbol{q}^{2}-i \varepsilon}}\right. \\
& \left.\quad+\int \varrho_{2}\left(m^{2}\right) \mathrm{d} m^{2} \frac{i}{2 \sqrt{m^{2}-\boldsymbol{q}^{2}}}\left[\frac{x}{n q-\sqrt{m^{2}-\boldsymbol{q}^{2}}+i \varepsilon}\left(-i \lambda_{2}^{\prime} \beta\right)_{\alpha \beta}\right)_{\alpha \beta}-\frac{\varkappa}{n q+\sqrt{m^{2}-\boldsymbol{q}^{2}-i \varepsilon}}\left(-i \lambda_{2}^{\prime} \beta\right)_{\alpha \beta}\right] .
\end{aligned}
$$

The spectral functions $\varrho_{i}\left(m^{2}\right)$ are:

$$
\begin{aligned}
& \varrho_{1}\left(m^{2}\right)=\delta\left(m^{2}-\varkappa^{2}\right)+\left(\varkappa^{2} / \omega^{2}-1\right) \delta\left(m^{2}\right)-\varkappa^{2} / \omega^{2} \delta\left(m^{2}-\omega^{2}\right)=: \sum_{i=1}^{3} \varrho_{1 i} \delta\left(m^{2}-\mu_{i}{ }^{2}\right) \\
& \varrho_{2}\left(m^{2}\right)=\delta\left(m^{2}-\varkappa^{2}\right)-\delta\left(m^{2}\right)=: \sum_{i=1}^{2} \varrho_{2 i} \delta\left(m^{2}-\mu_{i}{ }^{2}\right) .
\end{aligned}
$$

These spectral functions have been introduced in order to get a shorthand notation of the regularized twopoint function. The regularization is the usual one ${ }^{13}$ with the nucleon mass $\%$, but with the modification that we replace the dipole at mass zero by two poles at the masses $\omega$ and zero. 
The Green's function reads

$$
\tilde{G}_{\alpha \beta}(q)=\frac{1}{2 V-\boldsymbol{q}^{2}}\left[\frac{q_{\mu}}{n q-V-\boldsymbol{q}^{2}+i \varepsilon}\left(-\lambda_{2}^{\prime} \gamma^{\prime \mu} \beta\right)_{\alpha \beta}-\frac{q_{\mu}}{n q+V-\boldsymbol{q}^{2}-i \varepsilon}\left(-\lambda_{2}^{\prime} \gamma^{\prime \mu} \beta\right)_{\alpha \beta}\right] .
$$

The product of $F$ and $G$ consists of a sum of terms with the general form:

$$
n q-\sqrt{m^{2}-\boldsymbol{q}^{2}+i \varepsilon} \cdot \frac{1}{n q-V-\boldsymbol{q}^{2}+i \varepsilon} .
$$

These can be decomposed into partial fractions so that the eigenstates (4.9) only consist of terms with one denominator of the form $\left(\mathrm{n} q-\sqrt{m^{2}-\boldsymbol{q}^{2}}+i \varepsilon\right)$ as it must be for the spectral representation of any function $\varphi_{2}$.

The following computations will be done with the special timelike unit vector:

$$
n_{0}:=J / \sqrt{ } J^{2} .
$$

Due to the $\delta$-function $\delta\left(q_{1}+q_{2}-J\right)$ in (3.9) we can replace the variable $q_{1}$ by $\left(J-q_{2}\right)$ and vice versa. Having done this in some terms we finally get after some lengthy calculation our meson wave function:

$$
\begin{aligned}
& \tilde{\varphi}_{a_{1} \alpha_{2}}\left(q_{1} q_{2}\right)=l^{2}(3-I(\mathrm{I}+1)) \delta\left(q_{1}+q_{2}-J\right) \sum_{\lambda_{1} \lambda_{2}}(-1)^{\mathrm{P}} \int \mathrm{d} m^{2} \\
& \times\left\{\left[-Q_{\alpha_{\lambda_{1}} \alpha_{\lambda_{2}}}^{\rho \mu}\left(q_{{\dot{\lambda_{1}}}_{1}}\right)_{\varrho}\left(J-q_{{\dot{\lambda_{1}}}_{1}}\right)_{\mu} Q_{1}\left(m^{2}\right)+R_{\alpha_{\lambda_{1}} \alpha \lambda_{2}}^{o}\left(q_{\lambda_{1}}\right)_{2} \% Q_{2}\left(m^{2}\right)\right]\right. \\
& \times\left[\left(n_{0} q_{\lambda_{1}}-\sqrt{-\boldsymbol{q}_{\lambda_{1}}^{2}}+i \varepsilon\right)^{-1}\left(2 \sqrt{-\boldsymbol{q}_{\lambda_{1}}^{2}}\right)^{-1}\left(\left(n_{0} J-\sqrt{-\boldsymbol{q}_{\lambda_{1}}^{2}}\right)^{2}-\left(m^{2}-\boldsymbol{q}_{\lambda_{1}}^{2}\right)\right)^{-1}\right.
\end{aligned}
$$

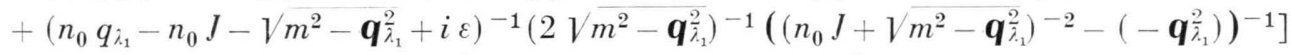

$$
\begin{aligned}
& +\left[Q_{\dot{\alpha} \lambda_{2} \alpha \lambda_{1}}^{o \mu}\left(J-q_{\lambda_{1}}\right)_{Q}\left(q_{\lambda_{\lambda_{2}}}\right)_{\mu} Q_{1}\left(m^{2}\right)-R_{\dot{\alpha} \lambda_{2} \alpha \lambda_{1}}^{o}\left(J-q_{\lambda_{1}}\right)_{Q} \varkappa Q_{2}\left(m^{2}\right)\right] \\
& \times\left[\left(n_{0} q_{\lambda_{1}}-n_{0} J-V-\boldsymbol{q}_{\lambda_{1}}^{2}+i \varepsilon\right)^{-1}\left(2 V-\boldsymbol{q}_{\lambda_{1}}^{2}\right)^{-1}\left(\left(n_{0} J+V-\boldsymbol{q}_{\lambda_{1}}^{2}\right)^{2}-\left(m^{2}-\boldsymbol{q}_{\lambda_{1}}^{2}\right)\right)^{-1}\right.
\end{aligned}
$$

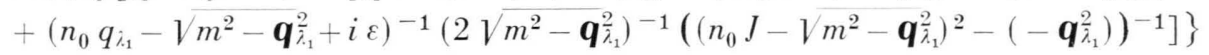

where we introduced the abbreviations:

$$
Q_{\alpha_{1} \alpha_{2}}^{o \mu}:=\left(-\lambda_{2}^{\prime} \gamma^{\prime} \varrho \beta\right)_{\alpha_{1} \alpha}\left[\left(\beta \lambda_{2}^{\prime} \tau^{\prime}\left(\mathrm{II}_{3}\right) \gamma^{\prime v}\right)^{\alpha \beta} J_{v} C^{\mathrm{II}_{3}, \mathrm{~V}}+\left(\beta \gamma_{5} \lambda_{2}^{\prime} \tau^{\prime}\left(\mathrm{II}_{3}\right) \gamma^{\prime}\right)^{\alpha \beta} \boldsymbol{J}_{v} C^{\mathrm{II}_{3}, \mathrm{~A}}\right]\left(-\lambda_{2}^{\prime} \gamma^{\prime \mu} \beta\right)_{\beta \alpha_{2}}
$$

and

$R_{\alpha_{1} \alpha_{2}}^{o}:=\left(-\lambda_{2}^{\prime} \gamma^{\prime} \varrho \beta\right)_{\alpha_{1} \alpha}\left[\left(\beta \lambda_{2}^{\prime} \tau^{\prime}\left(\mathrm{II}_{3}\right) \gamma^{\prime}\right)^{\alpha \beta} J_{\nu} C^{\mathrm{II}_{3}, \mathrm{~V}}+\left(\beta \gamma_{5} \lambda_{2}^{\prime} \tau^{\prime}\left(\mathrm{II}_{3}\right) \gamma^{\prime \prime}\right)^{\alpha \beta} J_{\nu} C^{\mathrm{II}_{3}, \mathrm{~A}}\right]\left(-i \lambda_{2}^{\prime} \beta\right)_{\beta \alpha_{2}}$.

If we look for the parity of the wave function, we can fix some of the constants. So by application of the parity operator ${ }^{13}$ to the wave function, we get:

$$
\left.C^{\mathrm{II}_{3}, \mathrm{~V}}=0 \quad \text { (for mesons with parity }-1\right)
$$

and

$$
\left.C^{\mathrm{II}_{3} \mathrm{~A}}=0 \quad \text { (for mesons with parity }+1\right) .
$$

Introducing an additional integration over $\boldsymbol{p}_{i}$ we obtain the proper spectral representation of the meson wave function $\varphi$.

$$
\tilde{\varphi}_{\alpha_{1} \alpha_{2}}\left(q_{1} q_{2}\right)=\Sigma \int_{i=1}^{4} \mathrm{~d}^{3} \boldsymbol{p}_{i} \mathrm{~d} m^{2} \Sigma\left({ }_{\lambda_{1} \lambda_{2}}-1\right)^{\mathrm{P}} \delta\left(q_{1}+q_{2}-J\right) M\left(m, p_{i} J\right) i(2 \pi)^{7} \delta\left(\boldsymbol{p}_{i}-\boldsymbol{q}_{\lambda_{1} \alpha_{\lambda_{2}}}\right)\left(n_{0} q_{\lambda_{1}}-n_{0} p_{i}+i \varepsilon\right)^{-1}
$$

comparable with (1.5) with the following four structure functions:

$$
\begin{aligned}
M\left(m, p_{1} J\right):=i & l^{2}(3-I(I+1))(2 \pi)^{7}\left[2 \sqrt{m^{2}-\boldsymbol{p}_{1}{ }^{2}}\left(\left(n_{0} J+\sqrt{m^{2}-\boldsymbol{p}_{1}{ }^{2}}\right)+\boldsymbol{p}_{1}{ }^{2}\right)\right]^{-1} \\
& \times\left[Q_{\alpha_{1} \alpha_{2}}^{\rho \mu} Q_{1}\left(m^{2}\right)\left(p_{1}\right)_{\varrho}\left(J-p_{1}\right)_{\mu}-R_{\alpha_{1} \alpha_{2}}^{o} \varrho_{2}\left(m^{2}\right)\left(p_{1}\right)_{\varrho} \varkappa\right]
\end{aligned}
$$

with

$$
q_{1}:=\left[J^{0}+\left(\left(p_{1}-\mathfrak{J}\right)^{2}+m^{2}\right)^{1 / 2}, p_{1}\right]
$$




$$
\begin{aligned}
M\left(m, \underset{\alpha_{1} \alpha_{2}}{p_{2}} J\right):=i & l^{2}(3-I(\mathrm{I}+1))(2 \pi)^{-7}\left[2 \sqrt{-\boldsymbol{p}_{2}{ }^{2}}\left(\left(n_{0} J-\sqrt{\left.-\boldsymbol{p}_{1}^{2}\right)^{2}}-\left(m^{2}-\boldsymbol{p}_{2}{ }^{2}\right)\right)\right]^{-1}\right. \\
& \times\left[Q_{\alpha_{1} \alpha_{2}}^{\rho \mu} \varrho_{1}\left(m^{2}\right)\left(p_{2}\right)_{\varrho}\left(J-p_{2}\right)_{\mu}-R_{\alpha_{1} \alpha_{2}}^{\varrho} \varrho_{2}\left(m^{2}\right)\left(p_{2}\right)_{\varrho} \varkappa\right]
\end{aligned}
$$

with

$$
p_{2}:=\left(\left|p_{2}\right|, p_{2}\right)
$$

$$
\begin{aligned}
M\left(m, p_{3} J\right): & =i l^{2}(3-I(I+1))(2 \pi)^{-7} \times\left[2 \sqrt{m^{2}-\boldsymbol{p}_{3}{ }^{2}}\left(\left(n_{0} J-\sqrt{m^{2}-\boldsymbol{p}_{3}{ }^{2}}\right)^{2}+\boldsymbol{p}_{3}{ }^{2}\right)^{-1}\right. \\
& \times\left[-Q_{\alpha_{2} \alpha_{1} \varrho_{1}}^{\rho \mu}\left(m^{2}\right)\left(J-p_{3}\right)_{\varrho}\left(p_{3}\right)_{\mu}+R_{\alpha_{2} \alpha_{1} \varrho_{2}}^{o}\left(m^{2}\right)\left(J-p_{3}\right)_{\varrho} \varkappa\right]
\end{aligned}
$$

with

$$
\begin{aligned}
p_{3}:=\left(\sqrt{m^{2}+p_{3}}, \mathfrak{p}_{3}\right) \\
M\left(m, p_{\alpha_{1} \alpha_{2}} J\right):= \\
\times\left[l^{2}(3-I(I+1))(2 \pi)^{-7} \times\left[2 \sqrt{-\boldsymbol{p}_{4}{ }^{2}}\left(\left(n_{0} J+\sqrt{\alpha_{2} \alpha_{1} \varrho_{1}}\left(m^{2}\right)\left(J-p_{4}\right)_{\varrho}\left(p_{4}\right)_{\mu}\right)^{2}-R_{\alpha_{2} \alpha_{1} \varrho_{2}}^{o}\left(m^{2}\right)\left(J-m_{4}\right)_{\varrho} \varkappa\right]\right.\right.
\end{aligned}
$$

with

$$
p_{4}:=\left(J^{0}+\left|p_{4}-\mathfrak{J}\right|, p_{4}\right)
$$

if one observes $\tau_{2} \equiv \varphi_{2}$ additionally.

\section{Normalization of Meson Eigenstates}

The physical scalar product in functional space is defined with the weighting operator ${ }^{6}$

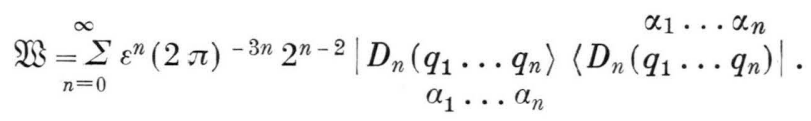

So we can form the physical scalar product

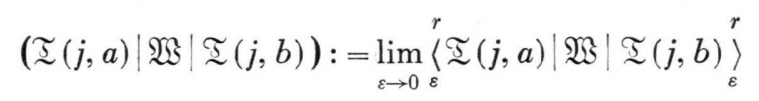

where the suffix $\mathrm{r}$ denotes the reduced functional ${ }^{6,7}$. As it was shown in earlier papers we can derive from (4.2) the formula:

$$
\langle\mathfrak{T}(j, a)|\mathfrak{W}| \mathfrak{T}(j, b)\rangle=\sum_{n=0}^{\infty} \frac{\varepsilon^{n}}{(n !)^{2}}(2 \pi)^{-3 n} 2^{n-2} \tilde{\tau}_{n}{ }^{*}\left(\begin{array}{c}
\alpha_{1} \ldots \alpha_{n} \\
\left(q_{1} \ldots q_{n} \mid a\right) \tilde{\tau}_{n}\left(q_{1} \ldots q_{n} \mid b\right) . \\
\alpha_{1} \ldots \alpha_{n}
\end{array}\right.
$$

In the special case, treated in this paper, the formula reads:

$$
\left\langle\mathfrak{T}\left(j, J^{\prime}\right)|\mathfrak{W}| \mathfrak{I}(j, J)\right\rangle=\frac{\varepsilon^{2}}{4}(2 \pi)^{-6} \tilde{\varphi}^{\alpha_{1} \alpha_{2}}\left(q_{1} q_{2} \mid J^{\prime}\right) \tilde{\varphi}_{\alpha_{1} \alpha_{2}}\left(q_{1} q_{2} \mid J\right) .
$$

Now we substitute the wave function (3.19) into the scalar product (4.4). As it was shown in ${ }^{7}$ only those terms give nonvanishing contributions which have the same permutations of the variables $q_{1}$ and $q_{2}$. This can be seen by performing the residual integration. Then for different permutations both poles lie in the same half plane of the complex $\left(n q_{i}\right)$-plane and their contributions vanish.

By a suitable change of the integration variables and the summation indices we get the formula:

$$
\begin{aligned}
& \left\langle\mathfrak{T}\left(j, J^{\prime}\right)|\mathfrak{W}| \mathfrak{T}(j, J)\right\rangle=\frac{\varepsilon^{2}}{4}(2 \pi)^{-6} 2 \sum_{j=1}^{4} \int \mathrm{d}^{3} \boldsymbol{p}_{j}^{\prime} \mathrm{d} m^{\prime 2} \delta\left(q_{1}+q_{2}-J^{\prime}\right) M^{*}\left(m^{\prime}, p^{\prime}{ }_{j}^{\alpha_{1}} J^{\prime}\right)(-i)(2 \pi)^{7} \\
& \quad \times \delta^{3}\left(\boldsymbol{p}_{j}^{\prime}-\boldsymbol{q}_{1}\right)\left(n_{0} q_{1}-n_{0} p_{j}^{\prime}-i \varepsilon\right)^{-1} \sum_{i=1}^{4} \int \mathrm{d}^{3} \boldsymbol{p}_{i} \mathrm{~d} m^{2} \delta\left(q_{1}+q_{2}-J\right) M\left(m, p_{\alpha_{1}} J\right) i(2 \pi)^{7} \\
& \quad \times \delta^{3}\left(\boldsymbol{p}_{i}-\boldsymbol{q}_{1}\right)\left(n_{0} q_{1}-n_{0} p_{i}+i \varepsilon\right)^{-1} .
\end{aligned}
$$


Now we perform the integration with respect to $q_{1}$ and $q_{2}$. The integration over $\left(n_{0} q_{1}\right)$ is a residual inle gration. This gives

$$
\begin{aligned}
& \left\langle\mathfrak{I}\left(j, J^{\prime}\right)|\mathfrak{W}| \mathfrak{I}(j, J)\right\rangle=\frac{\varepsilon^{2}}{2}(2 \pi)^{8} \sum_{j=1}^{4} \sum_{i=1}^{4} \int \mathrm{d}^{3} \boldsymbol{p}_{j}{ }^{\prime} \mathrm{d} m^{\prime 2} \mathrm{~d}^{3} \boldsymbol{p}_{i} \mathrm{~d} m^{2} \delta\left(J^{\prime}-J\right) \\
& \times M^{*}\left(m^{\prime}, p_{j}^{\alpha_{1} \alpha_{2}} J^{\prime}\right) M\left(m, p_{\alpha_{1} \alpha_{2}} J\right) \delta^{3}\left(\boldsymbol{p}_{j}{ }^{\prime}-\boldsymbol{p}_{i}\right) 2 \pi i\left(n_{0} p_{j}^{\prime}-n_{0} p_{i}+2 i \varepsilon\right)^{-1} .
\end{aligned}
$$

In order to demonstrate the further calculation procedure, we shall choose one term of the expression (4.6).

$$
\begin{aligned}
& I_{11}:=\frac{\varepsilon^{2}}{2}(2 \pi)^{8} \int \mathrm{d}^{3} \boldsymbol{p}_{1}{ }^{\prime} \mathrm{d} m^{\prime 2} \mathrm{~d}^{3} \boldsymbol{p}_{1} \mathrm{~d} m^{2} \delta\left(J-J^{\prime}\right) \delta^{3}\left(\boldsymbol{p}_{1}{ }^{\prime}-\boldsymbol{p}_{1}\right) 2 \pi i\left(n_{0} p_{1}{ }^{\prime}-n_{0} p_{1}+2 i \varepsilon\right)^{-1} \\
& \times(-i) l^{2}\left(3-I^{\prime}\left(I^{\prime}+1\right)\right)(2 \pi)^{-7}\left[2 \sqrt{m^{\prime 2}-\boldsymbol{p}_{1}^{\prime 2}}\left(\left(n_{0} J+\sqrt{m^{\prime 2}-p_{1}^{\prime 2}}\right)^{2}+\boldsymbol{p}_{1}^{\prime 2}\right)\right]^{-1} \\
& \times\left[Q^{*} \varrho^{\prime} \mu^{\prime} \alpha_{1} \alpha_{2} \sum_{j} \varrho_{1 j} \delta\left(m^{\prime 2}-\mu_{j}^{2}\right)\left(p_{1}^{\prime}\right)_{Q^{\prime}}\left(J^{\prime}-p_{1}{ }^{\prime}\right)_{\mu^{\prime}}-R^{*} \varrho^{\prime} \alpha_{1} \alpha_{2} \sum_{j} \varrho_{2 j} \delta\left(m^{\prime 2}-\mu_{j}{ }^{2}\right)\left(p_{1}{ }^{\prime}\right)_{\varrho} \varkappa\right] \\
& \times i l^{2}(3-I(I+1))(2 \pi)^{-7}\left[2 \sqrt{m^{2}-\boldsymbol{p}_{1}{ }^{2}}\left(\left(n_{0} J+\sqrt{m^{2}-\boldsymbol{p}_{1}{ }^{2}}\right)^{2}+\boldsymbol{p}_{1}{ }^{2}\right)\right]^{-1} \\
& \times\left[Q_{\alpha_{1} \alpha_{2}}^{\varrho \mu} \sum_{i} \varrho_{1 i} \delta\left(m^{2}-\mu_{i}^{2}\right)\left(p_{1}\right)_{\varrho}\left(J-p_{1}\right)_{\mu}-R_{\alpha_{1} \alpha_{2}}^{o} \sum_{i} \varrho_{2 i} \delta\left(m^{2}-\mu_{i}{ }^{2}\right)\left(p_{1}\right)_{e} \varkappa\right] .
\end{aligned}
$$

Now we make use of the fact that we had only a mass spectrum, generated by the discrete masses in the regularization terms of the two-point function. After an integration over $m^{2}$ and $m^{\prime 2}$ we obtain:

$$
\begin{aligned}
& I_{11}=\frac{\varepsilon^{2}}{2}(2 \pi)^{-6} \int \mathrm{d}^{3} \boldsymbol{p}_{1}{ }^{\prime} \mathrm{d}^{3} \boldsymbol{p}_{1} \delta\left(J-J^{\prime}\right) \delta^{3}\left(\boldsymbol{p}_{1}{ }^{\prime}-\boldsymbol{p}_{1}\right) \\
& \times \sum_{i j} 2 \pi i\left(n_{0} p_{1 j}{ }^{\prime}-n_{0} p_{1 i}+2 i \varepsilon\right)^{-1} l^{4}\left(3-I^{\prime}\left(I^{\prime}+1\right)\right)(3-I(I+1)) \\
& \times\left[2 \sqrt{j}^{2}-\boldsymbol{p}_{1}^{\prime 2}\left(\left(n_{0} J+\sqrt{\mu_{j}{ }^{2}-\boldsymbol{p}_{1}{ }^{\prime 2}}\right)^{2}+\boldsymbol{p}_{1}{ }^{\prime 2}\right)\right]^{-1}\left[2 \sqrt{\mu_{i}{ }^{2}-\boldsymbol{p}_{1}{ }^{2}}\left(\left(n_{0} J+\sqrt{\mu_{i}{ }^{2}-\boldsymbol{p}_{1}{ }^{2}}\right)^{2}+\boldsymbol{p}_{1}{ }^{2}\right)\right]^{-1} \\
& \times\left[Q^{*} \varrho^{\prime} \mu^{\prime} \alpha_{1} \alpha_{2} \varrho_{1 j}\left(p_{1 j}^{\prime}\right)_{Q^{\prime}}\left(J-p_{1 j}^{\prime}\right)_{\mu^{\prime}}-R^{*} \varrho^{\prime} \alpha_{1} \alpha_{2} \varrho_{2 j}\left(p_{1 j}^{\prime}\right)_{\varrho^{\prime}} \chi\right] \\
& \times\left[Q_{\alpha_{1} \alpha_{2}}^{o \mu} \varrho_{1 i}\left(p_{1 i}\right)_{Q}\left(J-p_{1 i}\right)_{\mu}-R_{\alpha_{1} \alpha_{2}}^{o} Q_{2 i}\left(p_{1 i}\right) \varkappa\right]
\end{aligned}
$$

where the fourmomentum $p_{1 i}$ is defined by

$$
n_{0} p_{1 i}:=n_{0} J+\sqrt{\mu_{i}^{2}-\boldsymbol{p}_{1}{ }^{2}} .
$$

Now we shall deal with the expression

$$
A(\varepsilon):=2 i \varepsilon\left(n_{0} p_{1 j}^{\prime}-n_{\mathbf{0}} p_{1 i}+2 i \varepsilon\right)^{-1}=2 i \varepsilon\left(\sqrt{\mu_{j}^{2}-\boldsymbol{p}_{1}^{\prime 2}}-\sqrt{\mu_{i}^{2}-\boldsymbol{p}_{1}{ }^{2}}+2 i \varepsilon\right)^{-1} .
$$

Due to the $\delta$-function $\delta^{3}\left(\boldsymbol{p}_{1}{ }^{\prime}-\boldsymbol{p}_{1}\right)$ we obtain in the limit $\varepsilon \rightarrow 0$

$$
A(\varepsilon)=\left\{\begin{array}{ll}
1 & \text { for } \mu_{j}=\mu_{i} \\
0 & \text { for } \mu_{j} \neq \mu_{i}
\end{array} .\right.
$$

This is the definition of the Kronecker $\delta$-symbol:

$$
\lim _{\varepsilon \rightarrow 0} A(\varepsilon)=\delta_{\mu_{j} \mu_{i}} .
$$

With this relation, we can perform the summation with respect to $j$ and the integral over $\boldsymbol{p}_{1}{ }^{\prime}$. Calculating the product of the matrices, we get:

$$
\begin{aligned}
& I_{11}=\frac{\varepsilon}{4}(2 \pi)^{-5} \int \mathrm{d}^{3} \boldsymbol{p}_{1} \delta\left(J-J^{\prime}\right) \sum_{i} l^{4}(3-I(I+1))^{2} \delta_{\mathrm{II}^{\prime}} \delta_{\mathrm{I}_{3} \mathrm{I}_{3}} \\
& \times\left[4\left(\mu_{i}{ }^{2}-\boldsymbol{p}_{1}{ }^{2}\right)\left(\left(n_{0} J+\sqrt{\mu_{i}^{2}-\boldsymbol{p}_{1}{ }^{2}}\right)-\boldsymbol{p}_{1}{ }^{2}\right)^{2}\right]^{-1} \\
& \times 2^{4}\left(\varrho_{1 i}{ }^{2}\left(p_{1}\right)^{2}\left(J-p_{1}\right)^{2}+\varrho_{2 i}{ }^{2}\left(p_{1}\right)^{2} x^{2}\right) J^{2}\left(\left|C^{\mathrm{II}_{3}, \mathrm{~V}}\right|^{2}-\left|C^{\mathrm{II}_{3}, \mathrm{~A}}\right|^{2}\right) .
\end{aligned}
$$

We introduce the new spectral functions

$$
\begin{gathered}
\bar{\varrho}_{1}\left(m^{2}\right):=\delta\left(m^{2}-\varkappa^{2}\right)+\left(\varkappa^{2} / \omega^{2}-1\right)^{2} \delta\left(m^{2}\right)+\left(\varkappa^{4} / \omega^{4}\right) \delta\left(m^{2}-\omega^{2}\right) \\
\bar{\varrho}_{2}\left(m^{2}\right):=\delta\left(m^{2}-\varkappa^{2}\right)+\delta\left(m^{2}\right)
\end{gathered}
$$


and with them, we obtain the following result:

$$
\begin{aligned}
I_{11}:= & \varepsilon(2 \pi)^{-5} l^{4}(3-I(I+1))^{2} \delta\left(J-J^{\prime}\right) \delta_{\mathrm{II}^{\prime}} \delta_{\mathrm{I}_{3} \mathrm{I}_{3}^{\prime}} \\
& \times \int \mathrm{d}^{3} \boldsymbol{p}_{1} \mathrm{~d} m^{2} J^{2}\left(\bar{\varrho}_{1}\left(m^{2}\right) m^{2}+\bar{\varrho}_{2}\left(m^{2}\right) \varkappa^{2}\right)\left(\left|C^{\mathrm{II}_{3}, \mathrm{~V}}\right|^{2}-\left|C^{\mathrm{II}_{3}, \mathrm{~A}}\right|^{2}\right) \\
& \times\left[\left(m^{2}-\boldsymbol{p}_{1}{ }^{2}\right)\left(\left(n_{0} J+\sqrt{\left.m^{2}-\boldsymbol{p}_{1}{ }^{2}\right)}\right)^{2}+\boldsymbol{p}_{1}{ }^{2}\right)\right]^{-1} .
\end{aligned}
$$

The other terms of the scalar product are computed in the same way. So we obtain:

$$
\begin{aligned}
& \left\langle\mathfrak{T}\left(j, J^{\prime}\right)|\mathfrak{W}| \mathfrak{I}(j, J)\right\rangle=\varepsilon(2 \pi)^{-5} l^{4}(3-I(I+1))^{2} \delta\left(J-J^{\prime}\right) \int \mathrm{d} m^{2} \mathrm{~d}^{3} \boldsymbol{p} J^{2}\left(\left|C^{\mathrm{II}}, \mathrm{V}\right|^{2}-\left|C^{\mathrm{II}_{3}, \mathrm{~A}}\right|^{2}\right) \\
& \times\left(\varrho_{1}\left(m^{2}\right) m^{2}+\bar{\varrho}_{2}\left(m^{2}\right) \varkappa^{2}\left(m^{2}-\boldsymbol{p}^{2}\right)^{-1}\left[\left(\left(n_{0} J+\sqrt{m^{2}-\boldsymbol{p}^{2}}\right)^{2}+\boldsymbol{p}^{2}\right)^{-1}+\left(\left(n_{0} J-\sqrt{m^{2}-\boldsymbol{p}^{2}}\right)^{2}+\boldsymbol{p}^{2}\right)^{-1}\right] .\right.
\end{aligned}
$$

The $\boldsymbol{p}$-integral is performed by taking the principal value at the pole.

$$
\begin{aligned}
\left\langle\mathfrak{T}\left(j, J^{\prime}\right)|\mathfrak{W}| \mathfrak{I}(j, J)\right\rangle=\varepsilon(2 \pi)^{-3} l^{2} \delta\left(J^{\prime}-J\right)(3-I(I+1))^{2} J^{2}\left(\left|C^{\mathrm{I}_{3}, \mathrm{~V}}\right|^{2}-\left|C^{\mathrm{I}_{3}, \mathrm{~A}}\right|^{2}\right) \\
\times \int \mathrm{d} m^{2}\left(\bar{\varrho}_{1}\left(m^{2}\right) m^{2}+\bar{\varrho}_{2}\left(m^{2}\right) \varkappa^{2}\right) \frac{-m}{J^{2}+m^{2}} .
\end{aligned}
$$

We use the formal relation, which has been introduced by Illig ${ }^{8}$

$$
\varepsilon \pi \delta\left(n_{0} J-n_{0} J\right)=1 .
$$

After the integration of the masses, we finally get the result:

$$
\begin{aligned}
\left(\mathfrak{I}\left(j, J^{\prime}\right)|\mathfrak{W}| \mathfrak{I}(j, J)\right)=(2 \pi)^{-4} 2 l^{4}(3-I(I+1))^{2} \delta^{3}\left(\boldsymbol{J}-\mathbf{J}^{\prime}\right) J^{2}( & \left.-\left|C^{\mathrm{II}, \mathrm{V}}\right|^{2}+\left|C^{\mathrm{II}_{\mathbf{s}}, \mathrm{A} \mid}\right|^{2}\right) \\
& \times\left(\frac{2 \varkappa^{3}}{J^{2}+\varkappa^{2}}+\frac{\varkappa}{\omega} \frac{\varkappa^{3}}{J^{2}+\omega^{2}}\right) .
\end{aligned}
$$

In this formula the positivity of the scalar product depends on the relation of the constants $C^{\mathrm{V}}$ and $C^{\mathrm{A}}$. As these constants are linked with the parity of the particle in consideration being a good quantum number in this version of the theory, either $C^{\mathrm{V}}$ or $C^{A}$ has to vanish. Therefore for particles with negative parity we have $C^{\mathrm{A}} \neq 0$ and $C^{\mathrm{V}} \equiv 0$, while for particles with positive parity we have $C^{\mathrm{A}} \equiv 0$ and $C^{\mathrm{V}} \neq 0$. In the first case the corresponding norm expression is positive, while in the second case it is negative. Now we postulate physical particles to have a positive norm. Then mesons with negative parity, namely the $\tau$ - and the $\eta$-meson are admitted, while spin zero mesons with positive parity are excluded. But this result corresponds just to the experimental facts. So already the functional normalization procedure gives reasonable effects to be compared with experiments. Finally a remark has to be made about the dependence of the normalization procedure on the ghost states used for the regularization of the theory. By (4.20) follows, that the normalization is only possible if the mass of one ghost-particle is unequal zero i.e. the transition to a dipole ghost regularization in this version of the theory is not possible. As Heisenberg proposed ${ }^{1}$ the ghost masses have to be identified with the lepton masses. In this case the transition to a dipole ghost appears to be an unphysical limit which is not physically meaningful and consequently is excluded by the functional normalization procedure. On the other hand if one likes to investigate dipole ghost regularizations on purely theoretical reasons, it might be that the introduction of an indefinite metric in functional space too ${ }^{3,4}$ enables to perform also a finite boson normalization. But this problem has to be discussed in further papers on the subject.

\section{Appendix I}

The functions $F$ and $G$ with invariant time ordering.

As we deal with the invariant time ordering of the field operators in this paper, we have to look for a representation of all functions, which we need for the calculation of the meson wave function to get an invariant time ordering. We shall do this for the two point function. The procedure for the Green's function is the same. 
The unregularized twopoint function of the Hermitean Dirac field in momentum space reads:

$$
\begin{aligned}
& \tilde{F}_{\alpha \beta}(q)=i \frac{q_{\mu}}{q^{2}-m^{2}+i \delta} \\
& \quad \times\left(-\lambda_{2}^{\prime} \gamma^{\prime \mu} \beta\right)_{\alpha \beta}+i \frac{m}{q^{2}-m^{2}+i \delta}\left(-i \lambda_{2}^{\prime} \beta\right)_{\alpha \beta} .
\end{aligned}
$$

As we have demonstrated in Section 2 all fourvectors can be decomposed into two parts, parallel and orthogonal to a given vector. So we can write for the square of momentum:

$$
q^{2}=(n q)^{2}+\boldsymbol{q}^{2} .
$$

In (I.3) we performed a decomposition in partial fractions where the variable is $(n q)$.

We then obtain the following form of the twopoint function:

$$
\begin{aligned}
\tilde{F}_{\alpha \beta}(q)= & \frac{i}{2 \sqrt{m^{2}-\boldsymbol{q}^{2}}} \frac{q_{\mu}}{n q-\sqrt{m^{2}-\boldsymbol{q}^{2}+i \varepsilon}} \\
& \cdot\left(-\lambda_{2}^{\prime} \gamma^{\prime \mu} \beta\right)_{\alpha \beta} \\
& -\frac{i}{2 \sqrt{m^{2}-\boldsymbol{q}^{2}}} \frac{q_{\mu}}{n q+\sqrt{m^{2}-\boldsymbol{q}^{2}}-i \varepsilon} \\
& \cdot\left(-\lambda_{2}^{\prime} \gamma^{\prime \mu} \beta\right)_{\alpha \beta} \\
& +\frac{i}{2 \sqrt{m^{2}-\boldsymbol{q}^{2}}} \frac{m}{n q-\sqrt{m^{2}-\boldsymbol{q}^{2}+i \varepsilon}} \\
& \cdot\left(-i \lambda_{2}^{\prime} \beta\right)_{\alpha \beta}
\end{aligned}
$$

1 W. Heisenberg, An Introduction to the Unified Theory of Elementary Particles, Wiley and Sons, London 1967.

2 H. P. Dürr, Quanten und Felder, Vieweg-Verlag, Braunschweig 1971, S. 125.

3 H. Stumpf, Acta Phys. Austr. Suppl. 9, 195 [1972].

4 H. Stumpf, Z. Naturforsch. 27 a, 1058 [1972].

5 H. Stumpf, Quanten und Felder, Vieweg-Verlag, Braunschweig 1971, S. 189.

${ }^{6}$ H. Stumpf, Z. Naturforsch. 26 a, 1730 [1971].

$$
\begin{aligned}
& -\frac{i}{2 \sqrt{m^{2}-\boldsymbol{q}^{2}}} \frac{m}{n q+\sqrt{m^{2}-\boldsymbol{q}^{2}}-i \varepsilon} \\
& \cdot\left(-i \lambda_{2}^{\prime} \beta\right)_{\alpha \beta} .
\end{aligned}
$$

Here we introduced

$$
\varepsilon=\delta / 2 \sqrt{m^{2}-\boldsymbol{q}^{2}} .
$$

The application of a fourier transformation results in the space representation:

$$
\begin{aligned}
F_{\alpha \beta}(x)= & (2 \pi)^{-3} \int \mathrm{d}^{4} q \Theta(n q) \delta\left(p^{2}-m^{2}\right) \\
& \times\left\{\Theta ( n x ) e ^ { - i q x } \left[q_{\mu}\left(-\lambda_{2}^{\prime} \gamma^{\prime \mu} \beta\right)_{\alpha \beta}\right.\right. \\
& \left.+m\left(-i \lambda_{2}^{\prime} \beta\right)_{\alpha \beta}\right]+\Theta(-n x) e^{i q x} \\
& \cdot\left[-q_{\mu}\left(-\lambda_{2}^{\prime} \gamma^{\prime \mu} \beta\right)_{\alpha \beta}\right. \\
& \left.\left.+m\left(-i \lambda_{2}^{\prime} \beta\right)_{\alpha \beta}\right]\right\} .
\end{aligned}
$$

Comparing this with the usually used twopoint function, one easily can see, that (I.5) has an invariant time ordering.

By the same considerations we can derive the formula for the Green's function. [One only has to take $m=0$ in (I.5).]

$$
\begin{aligned}
G_{\alpha \beta}(q)= & \frac{1}{2 \sqrt{-\boldsymbol{q}^{2}}} \frac{q_{\mu}}{n q-\sqrt{-\boldsymbol{q}^{2}}+i \varepsilon} \\
& \cdot\left(-\lambda_{2}^{\prime} \gamma^{\prime \mu} \beta\right)_{\alpha \beta} \\
& -\frac{1}{2 V-\boldsymbol{q}^{2}} \frac{q_{\mu}}{n q+\sqrt{-\boldsymbol{q}^{2}-i \varepsilon}} \\
& \cdot\left(-\lambda_{2}^{\prime} \gamma^{\prime \mu} \beta\right)_{\alpha \beta} .
\end{aligned}
$$

7 D. Englert, Rep. Math. Phys. (in press).

8 K. Illig, Z. Naturforsch. 29 a, 1 [1974].

9 G. Schäfer, Master thesis (unpublished).

10 H. P. Dürr and F. Wagner, Nuovo Cim. 46, 223 [1966].

11 D. A. Nutbrown, Phys. Rev. D 3, 2981 [1971].

12 K. Dammeier, Z. Naturforsch. 27 a, 1042 [1972].

13 J. D. Bjorken and S. D. Drell, Relativistic Quantum Mechanics, McGraw Hill, New York. 